\title{
A New Approach for Finding the Initial Solution of the Unbalanced Transportation Problem
}

\author{
Qazi Shoeb Ahmad \\ ${ }^{1}$ Department of Statistics, \\ Science Unit, Deanship of Educational Services, \\ Qassim University, Buraidah, KSA \\ Email:qazishoeb@gmail.com
}

\begin{abstract}
A transportation problem deals with two different problems balanced transportation problem or unbalanced transportation problem. This paper points out how Goyal's modification of Vogel's approximation method for the unbalanced transportation problem can be improved by subtracting or adding suitable constants to the cost matrix, rows and columns of the cost matrix. In this paper, a new method is proposed for solving unbalanced transportation problem which gives optimal or very near to optimal solution.
\end{abstract}

Keywords - Transportation problem, unbalanced transportation problem, initial basic feasible solution, Vogel's approximation method

\section{INTRODUCTION}

The transportation problem constitutes an important part of logistics management. If in a transportation problem, the sum of supply available from all sources is not equal to the sum of demands of all destinations, i.e. the problem is said to be unbalanced transportation problem. The unbalanced transportation problem is a particular class of transportation problem, which is associated with day-to-day activities in our real life and mainly deals with logistics. It helps in solving problems on distribution and transportation of resources from one place to another. The goods are transported from " $\mathrm{m}$ " sources to "n" designations and their capacities are $a_{1}, a_{2}, \ldots, a_{m}$ and $b_{1}, b_{2, \ldots}, b_{n}$ respectively. In addition, there is a penalty $c_{i j}$ associated with transporting unit of product from source $i$ to destination $j$. This penalty may be cost or delivery time or safety of delivery etc. A variable $x_{i j}$ represents the unknown quantity to be shipped from source $i$ to destination $j$. The objective is to satisfy the demand at destinations from the supply constraints at the minimum transportation cost.

There are various heuristic methods available to get an initial basic feasible solution, such as North West Corner rule, least cost method, Vogel's approximation method (VAM), (Reinfeld and Vogel, 1958), Goyal's version of VAM (1984). Further, Kirca and Satir (1990) developed a heuristic, called TOM (Total Opportunity-cost Method), for obtaining an initial basic feasible solution for the transportation problem. Q. S. Ahmad (2008) proposed a new approach for initial solution of transportation problem, Sharma and Sharma (2000) proposed a new heuristic approach for getting good starting solutions for dual based approaches used for solving transportation problems. C. S. Ramakrishnan (1988) proposed a method for the unbalanced transportation problem.

\section{VAM and Goyal's Method:}

VAM (Vogel's approximation method) ${ }^{8}$ for a balanced transportation problem starts with the calculation of penalties (the difference between the second minimum and the first minimum costs) for all rows and columns. Then it allocates as many units as possible to the least cost cell in the row or column having maximum penalty. Then the allocated row/column is deleted, penalties are revised and the procedure repeated successively until all units are supplied. This paper is self-contained. Goyal ${ }^{4}$ gives more details, and K.G. Murthy's book ${ }^{6}$ is recommended.

It is well known that an unbalanced transportation problem is equivalent to an ordinary balanced transportation problem with one (dummy) column or row with zero costs added. Because of these zeros, VAM applied to this problem does not yield satisfactory results. To overcome this difficulty, Goyal ${ }^{4}$ suggested replacing the zero cost in dummy column by the largest unit transportation cost. We now reproduce his example and solution. 
Suppose we have the following transportation problem:

\begin{tabular}{|c|c|c|c|c|}
\hline & 1 & 2 & 3 & Supplies \\
\hline $\mathrm{A}$ & 6 & 10 & 14 & 50 \\
$\mathrm{~B}$ & 12 & 19 & 21 & 50 \\
$\mathrm{C}$ & 15 & 14 & 17 & 50 \\
\hline Demand & 30 & 40 & 55 & $125 \neq 150$ \\
\hline
\end{tabular}

Clearly the above problem is unbalanced transportation problem, to make the balanced transportation problem one has to introduce dummy column. After adding the dummy column, the problem is as follows:

\begin{tabular}{|c|c|c|c|c|c|}
\hline & 1 & 2 & 3 & 4 (Dummy) & Supplies \\
\hline $\mathrm{A}$ & 6 & 10 & 14 & 0 & 50 \\
$\mathrm{~B}$ & 12 & 19 & 21 & 0 & 50 \\
$\mathrm{C}$ & 15 & 14 & 17 & 0 & 50 \\
\hline Demand & 30 & 40 & 55 & 25 & 150 \\
\hline
\end{tabular}

If we replace the zeros in the dummy column by 21 (the largest transportation cost) and apply VAM, we get Goyal's solution as:

B to 1 (30), A to 2 (40), A to 3 (10), C to 3 (45), B to 4 (20) and C to 4 (5)

The value of the objective function $Z=1665$. We now suggest a modification of Goyal's method which yields the optimal cost of 1650 in his example.

\section{PROPOSED METHOD}

Our method consists of four steps of reduction and one step of VAM as follows:

Step 1: Subtract the minimum element of the original matrix from all the elements of the matrix.

Step 2: Now introduce dummy row or column and write the maximum element of new matrix in dummy row or column.

Step 3: Subtract minimum element of each row.

Step 4: Subtract minimum element of each column.

Step 5: Then apply VAM to the reduced matrix.

The first step applied to the Goyal's example gives the following cost matrix:

\begin{tabular}{|c|c|c|c|c|}
\hline & 1 & 2 & 3 & Supplies \\
\hline $\mathrm{A}$ & 0 & 4 & 8 & 50 \\
$\mathrm{~B}$ & 6 & 13 & 15 & 50 \\
$\mathrm{C}$ & 9 & 8 & 11 & 50 \\
\hline Demand & 30 & 40 & 55 & $125 \neq 150$ \\
\hline
\end{tabular}

The second step applied to the above cost matrix gives the following matrix:

\begin{tabular}{|c|c|c|c|c|c|}
\hline & 1 & 2 & 3 & 4 (Dummy) & Supplies \\
\hline $\mathrm{A}$ & 0 & 4 & 8 & 15 & 50 \\
$\mathrm{~B}$ & 6 & 13 & 15 & 15 & 50 \\
$\mathrm{C}$ & 9 & 8 & 11 & 15 & 50 \\
\hline Demand & 30 & 40 & 55 & 25 & 150 \\
\hline
\end{tabular}

The third and fourth step applied to the above cost matrix gives the following matrix:

\begin{tabular}{|c|c|c|c|c|c|}
\hline & 1 & 2 & 3 & 4 (Dummy) & Supplies \\
\hline A & 0 & 4 & 5 & 8 & 50 \\
B & 0 & 7 & 6 & 2 & 50 \\
C & 1 & 0 & 0 & 0 & 50 \\
\hline Demand & 30 & 40 & 55 & 25 & 150 \\
\hline
\end{tabular}

Now applying step 5 (VAM) to the above reduced cost matrix, we get the optimal solution to the original problem as follows:

with objective function value $=1650$.

A to $1(5)$, A to $2(40)$, A to $3(5), B$ to $1(25), \mathrm{B}$ to $4(25), \mathrm{C}$ to $3(50)$

\section{CONCLUSIONS}

The proposed method works with smaller integers and the final matrix has a zero in each row/column - a condition satisfied by the optimal simplex (primal-dual) tableau ${ }^{6}$. Further, it gives optimal solution in most of the cases or it gives 
very near to optimal solution. So, less iterations are required to achieve the optimality in comparison to the initial solution by VAM and then optimality.

\section{REFERENCES}

[1] Ahmad Q. S., "A new approach for initial solution of transportation problem", The Manager, ISSN: 0973-8231, Vol. 3, No.1, 65-66, January-June, 2008.

[2] Dantzig, G.B., Linear Programming and Extensions, Princeton, NJ: Princeton University Press, 1963.

[3] Goyal S. K., "A note on a heuristic for obtaining an initial solution for the transportation problem", Journal of Operational Research Society, Vol. 42(9), 819-821, 1991.

[4] Goyal S. K., "Improving VAM for unbalanced transportation problems", Journal of Operational Research Society, Vol. 35, pp. 1113-1114, 1984.

[5] Kirca O. and Satir A., "A heuristic for obtaining an initial solution for the transportation problem", Journal of Operational Research Society, Vol. 41(9), pp. 865-871, 1990.

[6] Murthy K. G., Linear and Combinatorial Programming, Wiley (Inter Science), New York, 1976

[7] Ramakrishnan C. S., "An improvement to Goyal's modified VAM for the unbalanced transportation problem", Journal of Operational Research Society, Vol. 39, pp. 609-610, 1988.

[8] Reinfeld N. V. and Vogel W. R., Mathematical Programming, pp. 59-70. Prentice Hall, Englewood Cliffs, N.J., 1958

[9] Sharma, R. R. K. and K. D. Sharma, "A new dual based procedure for the transportation problem", European Journal of Operational Research, Vol.122 (3), pp. 611-624, 2000. 